\title{
Predicting aerodynamic resistance of brush seals using computational fluid dynamics and a 2-D tube banks model ${ }^{\text {is }}$
}

\author{
Yuchi Kang ${ }^{\mathrm{a}, \mathrm{b}}$, Meihong Liu, ${ }^{\mathrm{a}, *}$, Sharon Kao-Walter ${ }^{\mathrm{b}, \mathrm{c}}$, Wureguli Reheman ${ }^{\mathrm{b}}$, Jinbin Liu ${ }^{\mathrm{a}}$ \\ a Faculty of Mechanical and Electrical Engineering, Kunming University of Science and Technology, Kunming, China \\ ${ }^{\mathbf{b}}$ Department of Mechanical Engineering, Blekinge Institute of Technology, Karlskrona, Sweden \\ ${ }^{\mathrm{c}}$ College of Engineering, Shanghai Polytechnic University, Shanghai, China
}

\section{A R T I C L E I N F O}

\section{Keywords:}

Brush seal

Aerodynamic resistance

Euler number

Reynolds number2010 MSC:

00-01

99-00

\begin{abstract}
A B S T R A C T
Aerodynamic resistance of a brush seal was mainly studied. The velocity distribution along three specified lines was presented. By considering the pressure differential, Reynolds number and Euler number $(E u)$ were modified. The effect of geometric arrangements and pressure differentials on Eu and leakage were analyzed. Two correlations were fitted based on the numerical results. The results reveal the velocity distribution is almost flat, asymmetric along the specified lines. The velocity increases and decreases almost linearly at centerlines. $E u$ decreases gradually less with the increase of pressure differential and trends towards a fixed value. A larger $E u$ indicates stronger resistance but not necessarily less leakage. Finally, two fitted correlations are developed and one is exponential to the row number fits better.
\end{abstract}

\section{Introduction}

A brush seal, a novel type contact seal, has been widely used in recent decades. It is characterized by excellent leakage performance, low friction and rotor excursion capability. A typical brush seal consists of a back plate, a front plate and thousands of bristles, as illustrated in Fig. 1. Generally, a brush seal is used to reduce parasitic leakage between turning and stationary components of a turbomachinery.

Many literature have been focused on numerical simulation to analyze fluid flow in brush seals. One numerical model for assessing brush seals is the tube bank model, which contains in-line or staggered arranged circles. Dai and Liu developed a compact, staggered tube array numerical model and analyzed resistance which revealed an exponential relationship between energy loss and Reynolds number. In addition, the extent of resistance increased dramatically with a more compact geometry [1]. Huang et al. analyzed the fluid flow in a brush seal based on a two-dimensional (2-D) staggered tube bundle model. They argued that the sealing effect can be significantly enhanced by reducing the inter-tube spacing of the bristles for normal pressure differentials and the number of axial bristle rows [2]. Tan et al. proposed a meshing method which is able to achieve a better mesh independence and studied the separation points in tube banks models of a brush seal. They found that the separation point may move to the rear part of the bristle when the brush seal is subjected to a high pressure differential
[3]. Wang et al. analyzed the flows of two-stage brush seals by staggered tube bank model [4].

In addition to analyze flow across tube banks in the brush seal, several studies over the years have adopted tube bank models in heat exchangers. Aerodynamic or hydraulic resistance is one of the primary quantities of heat exchangers and is characterized by the total pressure drop. This is a form of energy loss partially caused by neighboring tubes and service conditions. Jin et al. performed numerical studies about the pressure drop in H-type finned tube banks with 10 rows and presented the correlation of Euler number $(\mathrm{Eu})$ for the 10-row tube banks model [5]. Chen et al. analyzed the relationship between pressure drop and geometric parameters. The predictable correlations of $E u$ were developed based on the experimental results [6]. Studying the pressure loss of tube banks with bimetallic fins, Kuntysh et al. found that tight-finned tube banks have better thermal efficiency than a loose one [7]. The study about the pressure drop in tube banks has been conducted in terms of geometry parameters such as longitudinal and transverse pitch ratios and working conditions such as Reynolds number.

A brush seal is used for secondary system sealing performance in a gas turbine, where a higher pressure ratio can improve global efficiencies. So the pressure differential for a brush seal is a key design factor. According to the literature, brush seal leakage increases exponentially with excessive pressure loads [9]. Bristle deformation, pressure stiffening effect, and hysteresis are associated with the

\footnotetext{
Fully documented templates are available in the elsarticle package on CTAN.

* Corresponding author.

E-mail address: 64115737@qq.com (M. Liu).
} 


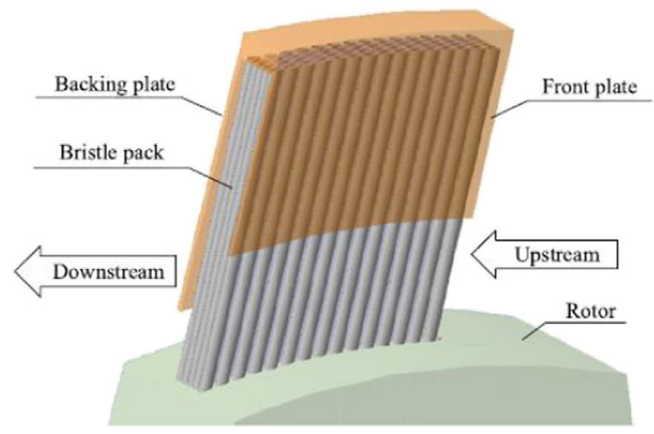

Fig. 1. Schematic diagram of a typical brush seal [8].

pressure differential across a brush seal. Zhu argued that $0.3 \sim 0.35 \mathrm{MPa}$ is the maximum load for these seals [10]. By contrast, Chupp et al. reported that the typical operation limit is $0.21 \mathrm{MPa}$ [11].

A review of the literature presents that almost all studies about brush seal pressure capability are based on experience. Although no model can be comprehensive enough to be applicable for all geometric arrangements, a few cases can be used to test a hypothesis. A quantity, $\mathrm{Eu}$, has been used to express the aerodynamic resistance of tube banks in heat exchangers. Therefore, to analyze the aerodynamic resistance of the brush seal, $E u$ is adopted in the present study. In addition, the effect of parameters, such as longitudinal and transverse pitch ratios, and pressure differentials, on aerodynamic resistance are studied. Furthermore, the correlation of $E u$ is developed based on numerical results. The results of this study can provide a theoretical basis for measuring resistance in brush seals and serve as a reference for industrial application.

\section{Model description and numerical method}

\subsection{Physical model}

A schematic diagram of a multiple-row brush seal tube bank model is presented in Fig. 2. The geometric parameters of the tube bank model are listed in Table 1.The geometry in the present study is by refering Huang's aforementioned model [2]. They found that the 1-column model and the 6-column model are equivalent for analyzing intertube flow.

To simplify the expression, $(A, B, C)_{p}^{N}$ stands for the different cases according to the geometric parameters in Table 1 . A, B and C stands for the angle $\theta$ of $30^{\circ}, 45^{\circ}, 60^{\circ}$, respectively, where subscript $p$ is the pressure differential and superscript $N$ is the total row number of bristles in a brush seal. For example, $A_{0.1}^{10}$ denotes the case $\theta=30^{\circ}$, pressure differential 0.1 MPa and row number 10 .

\subsection{Governing equations and boundary conditions}

Before starting a computational fluid dynamics (CFD) numerical simulation, the flow status must be assessed. Previous studies reveal different opinions about the flow status in a brush seal. Some researchers believe that the fluid flow is turbulent $[3,8,12]$ while others believe it becomes laminar. Huang et al. adopted the laminar model in compact tube banks and the turbulent model in spacious tube banks $[2,13]$. Zhang et al. argued that fluid flow is turbulent in the upstream
Table 1

Geometric parameters of a brush seal based on tube banks model.

\begin{tabular}{llllllll}
\hline Case & $\theta /{ }^{\circ}$ & $S_{D} / d$ & $S_{L} / d$ & $S_{T} / d$ & $\delta / \mathrm{mm}$ & $\mathrm{d} / \mathrm{mm}$ & $\mathrm{N}$ \\
\hline $\mathrm{A}$ & 30 & 1.1 & 0.953 & 1.1 & 0.007 & 0.07 & $10,14,18$ \\
$\mathrm{~B}$ & 45 & 1.1 & 0.778 & 1.556 & 0.007 & 0.07 & $10,14,18$ \\
$\mathrm{C}$ & 60 & 1.1 & 0.55 & 1.905 & 0.007 & 0.07 & $10,14,18$ \\
\hline
\end{tabular}

and downstream regions but becomes laminar in the intertube due to slow fluid flow [14]. According to the states of flow around a circular cylinder in different flow regimes, the transition (from laminar to turbulent) occurs at low Reynolds number [15]. A turbulent model was adopted in this research.

The fluid was assumed to be an ideal gas in a steady state. The governing equations of this research includes those of mass, momentum and energy conservation, all of which have been previously described elsewhere. The turbulent model is presented below. The standard $k-\varepsilon$ turbulent model was used and expressed as

$\rho \frac{D k}{D t}=\frac{\partial}{\partial x_{i}}\left[\left(\mu+\frac{\mu_{t}}{\sigma_{k}}\right) \frac{\partial k}{\partial x_{i}}\right]+G_{k}+G_{b}-\rho \varepsilon-Y_{M}$

$\rho \frac{D \varepsilon}{D t}=\frac{\partial}{\partial x_{i}}\left[\left(\mu+\frac{\mu_{t}}{\sigma_{k}}\right) \frac{\partial \varepsilon}{\partial x_{i}}\right]+C_{1 \varepsilon} \frac{\varepsilon}{k}\left(G_{k}+C_{3 \varepsilon} G_{b}\right)-C_{2 \varepsilon} \rho \frac{\varepsilon^{2}}{k}$

where $G_{k}$ is the formation of turbulent kinetic energy because of the average velocity gradients, $G_{b}$ is the formation of turbulent kinetic energy due to buoyancy, and $Y_{M}$ is the contribution of the fluctuating dilatation on compressible turbulence to the overall dissipation rate. $\mu_{t}$ is the eddy viscosity, which was modeled as $\mu_{t}=\rho C_{\mu} \frac{k^{2}}{\varepsilon}$ with $C_{\mu}=0.0845$. The standard model yields $C_{1 \varepsilon}=1.44, C_{2 \varepsilon}=1.92, C_{\mu}^{\varepsilon}=0.09$, $\sigma_{k}=1.0, \sigma_{\varepsilon}=1.3[16]$.

To guarantee inlet uniformity, the upstream domain was extended by fifteen times of the bristle diameter for the entrance section. To reduce the effect of reverse flow, the downstream domain was extended by twenty times of the bristle diameter for the exit section.

There are four different boundary conditions in this model. At the inlet boundary, pressured air enters the computational domain with the turbulent intensity 5\%. The outlet boundary pressure was set as $0.101 \mathrm{MPa}$. The bristle surface was assumed to be fixed and set as wall. The upper surface and lower surface were set as symmetric to simulate a more authentic flow domain. A detailed description of these boundary condition are presented in Table 2.

2-D steady-state numerical simulations were solved by commercial code FLUENT. A hybrid discretization scheme (the default settings in FLUENT) was used until a preliminary convergence is obtained. Then the second-order discretization scheme was adopted to gain a better solution efficiency. The residual criteria for each variable was $1.0 \times 10^{-6}$, except for continuity. By monitoring values between iterations is another criterion of the FLUENT tutorial [17]. The velocity difference, between different iteration steps at Line $\mathrm{CD}$ was monitored (Fig. 2).

\subsection{Grid meshing and independent solution}

By reviewing the previous method [3], an inflation tool was used to generate four layers of quadrilateral cells in contact with bristle

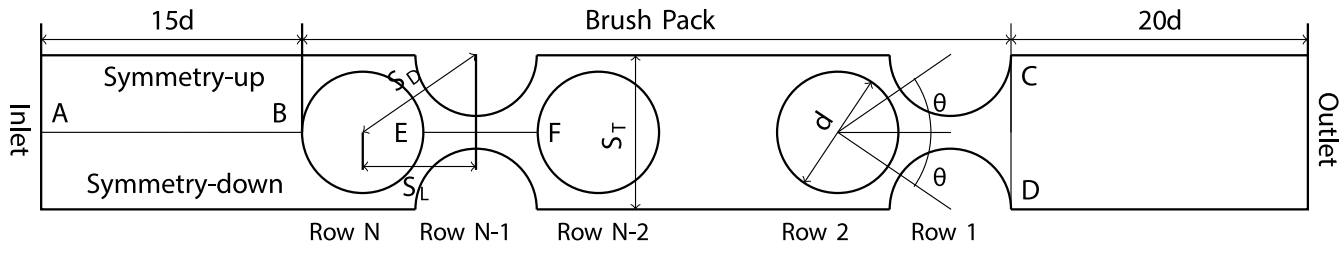

Fig. 2. A schematic configuration of a brush seal using a two-dimensional tube banks model. 
Table 2

Boundary conditions of the model.

\begin{tabular}{lllll}
\hline Location & Type & $\begin{array}{l}\text { Axial } \\
\text { Velocity } \\
(\mathrm{m} / \mathrm{s})\end{array}$ & $\begin{array}{l}\text { Circumferential } \\
\text { Velocity }(\mathrm{m} / \mathrm{s})\end{array}$ & Pressure (MPa) \\
\hline Inlet & Pressure & & 0.2010 .231 \\
& & & 0.2610 .291 \\
Outlet & Pressure & & 0.3210 .351 \\
Upper Wall & Symmetry & $\frac{\partial u}{\partial y}=0$ & 0 & 0.101 \\
Bottom Wall & Symmetry & $\frac{\partial u}{\partial y}=0$ & 0 & \\
Bristle & Wall & 0 & 0 & \\
\hline
\end{tabular}

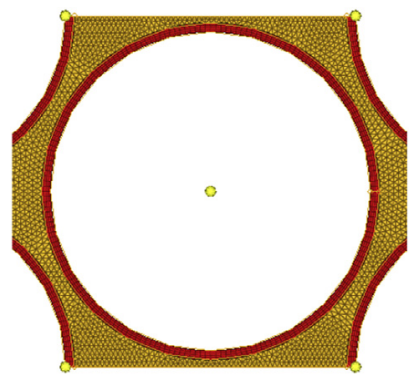

Fig. 3. An illustration of the generated mesh.

surfaces. The domains of the upstream and downstream regions meshed into quadrilateral cells. The rest of the domains were meshed into triangular cells. Part of the mesh is presented in Fig. 3. Five different meshing from coarse to fine were generated so as to investigate the grid-independency of the solution, including approximately $2.09 \times 10^{5}$, $2.53 \times 10^{5}, 3.02 \times 10^{5}, 3.39 \times 10^{5}$ and $3.6 \times 10^{5}$ cells. The velocity along Line $\mathrm{CD}$ was used to investigate mesh independence, because velocity changes obviously at Line $\mathrm{CD}$. Generally considering the mesh independence and computational time, the grid amount of $3.02 \times 10^{5} \mathrm{can}$ be viewed as grid independent.

\subsection{Validation of the numerical model}

To validate the reliability of the computational model and numerical method, a comparison between the numerical result and experimental or analytical results should be done. The brush seal experiments by Bayley and Long are frequently cited in the literature [2,18]. Twenty-four pressure tappings were adopted, eleven of which were expected to be under the bristle tips, in the rotor surface. The rest 13 pressure tappings were designed to accommodate the predicted axial deflection of the bristles and other uncertainty. Experiments were conducted at upstream absolute pressures ranging from $107 \mathrm{KPa}$ to $380 \mathrm{KPa}$, and a downstream absolute pressure of $100 \mathrm{KPa}$. The effect of rotation was not considered [19]. Cases $A_{0.1}^{10}$ and $A_{0.19}^{10}$ were used to compare with the experimental results. Fig. 4 represents the validation of the current model. The pressure distribution along centerlines at each gap was compared with the experimental results. The centerlines are described as Line EF in Fig. 2. The $X^{*}$ and $P^{*}$ denotes two dimensionless variables, which are expressed as

$X^{*}=\frac{x-x_{u}}{x_{d}-x_{u}}$

$P^{*}=\frac{p-p_{d}}{p_{u}-p_{d}}$

where $x_{u}$ is the $\mathrm{x}$ coordinate of the end of the first bristle gap centerline, and $x_{d}$ is the $\mathrm{x}$ coordinate of the beginning of the last bristle gap centerline. Parameters $p_{u}$ and $p_{d}$ are the pressure upstream and downstream of the brush seal, respectively. Fig. 4 presents the numerical

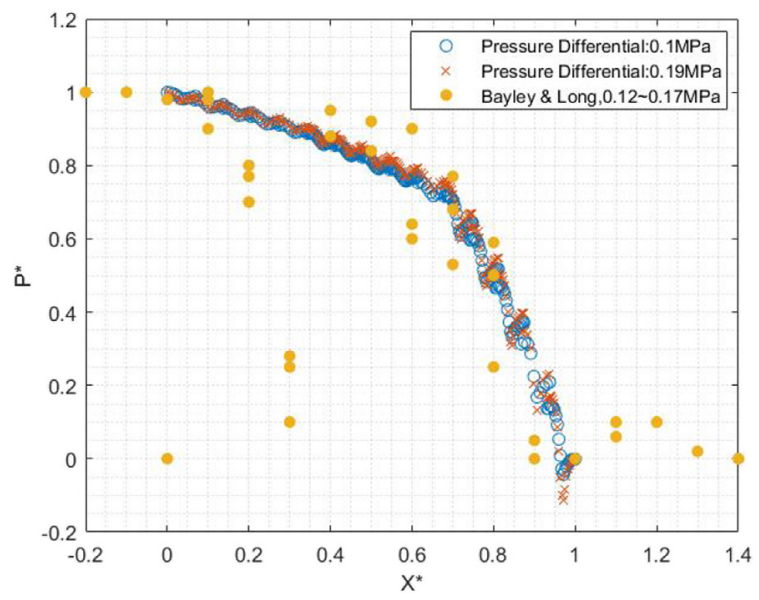

Fig. 4. A comparsion of pressure distribution between the numerical and experiemental results.

results, which reveal a trend similar to that of experimental results. Although a slight difference between the two results, their similarity confirms the validity of the model to some extents.

\section{Numerical results and discussion}

\subsection{Velocity distribution}

Fluid velocity distribution is a basic analysis for flow in a tube banks model. Fig. 5 shows the velocity distribution along Line $\mathrm{AB}$ and Line $\mathrm{CD}$ (in Fig. 2). The $x$-axis represents the date counts from the post-processing software. The y-axis represents the dimensionless velocity, defined as the velocity divided by the maximum. In Line $\mathrm{AB}$, the velocity is almost flat but then decreases to zero when approaching the bristle surface. In addition, the velocity is larger for a high pressure differential than a low pressure differential. In Line $\mathrm{CD}$, the velocity is almost symmetrical for Case $B_{10}^{0.16}$ and Case $B_{10}^{0.1}$. However, the velocity distribution is asymmetrical for Case $B_{10}^{0.22}$.

This asymmetrical distribution is deemed as the bistable phenomenon. It can be characterized by two vortices: a narrow near-wake region at the rear part of one of the cylinders, and a wide near-wake at the rear part of the other cylinder. Fig. 6 demonstrates the scheme of two bistable flow modes after two circles in-line arranged. Rather than being triggered by the arrangement of the cylinders or other external factors, these bistable characteristics are an intrinsic feature of the flow [20]. Fig. 7 presents the velocity distribution at Line CD for Case $(A, B, C)_{14}^{0.1}$, which clearly reveals the symmetrical velocity distribution

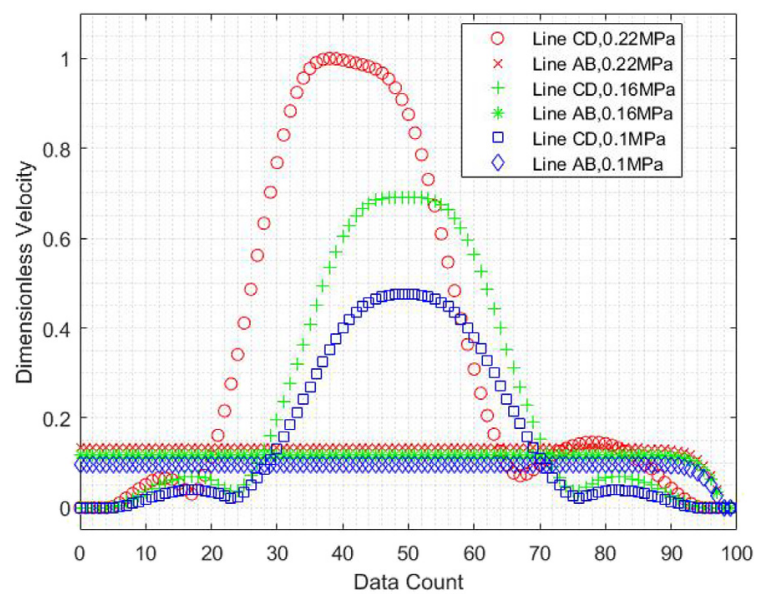

Fig. 5. Velocity distribution at line $\mathrm{AB}$ and $\mathrm{CD}$ for Case $B_{10}$. 


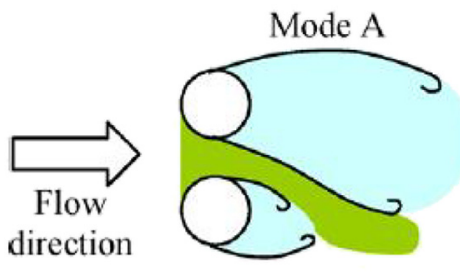

(a)

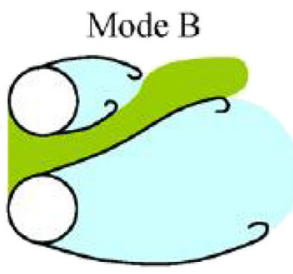

(b)
Fig. 6. Schematic diagram of two bistable flow models after two circles side by side [21].

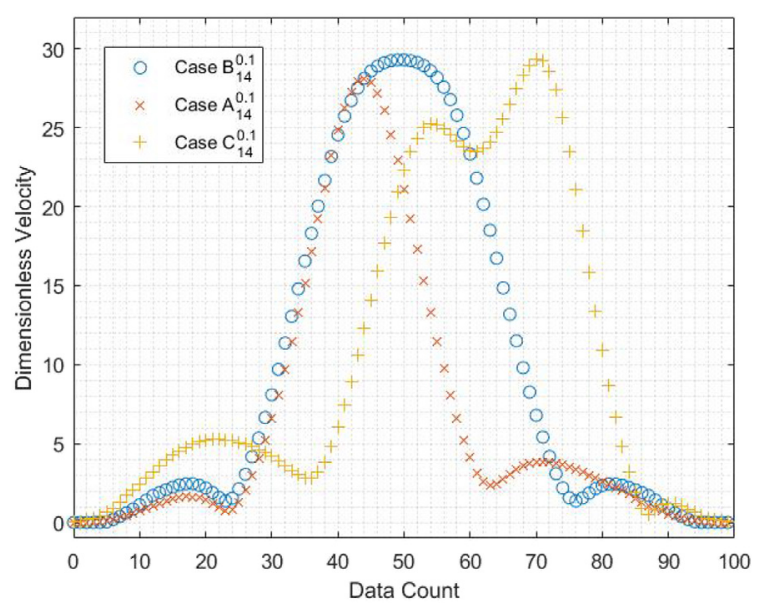

Fig. 7. Velocity distribution at Line $\mathrm{AB}$ for Case $(A, B, C)_{14}^{0.1}$.

for Case A and Case C. In addition, this distribution contained two peaks in Case $C_{14}^{0.1}$. This topic of bistable phenomenon requires further attention but it is beyond the scope of the present study.

The velocity inside the bristle pack is analyzed here to calculate Reynolds number in a brush seal. For each case, the velocity distribution along the centerlines of the narrowest gap is viewed and analyzed. As illustrated in Fig. 8, the geometry from left to right is for Case A, Case B and Case C, respectively. The velocities along Line AB, Line CD and Line EF were extracted for Case $A$. Line GH and $\mathrm{HI}$ for the Case $B$. Line JK and LM for the Case $C$. Velocity varies much more significantly along these centerlines than along the centerlines of the spacious gaps, which indicates velocity along centerlines of the narrowest gaps is more representative.

Fig. 9 presents the velocity distribution along Line GH and Line $\mathrm{HI}$ for Case $B_{10}^{0.1}, B_{10}^{0.16}$ and $B_{10}^{0.22}$. Velocity distribution along Line $\mathrm{AB}$, Line $\mathrm{CD}$ and Line EF for Case A and Line JK and Line LM for Case C are similar to Line GH and Line HI for Case B in trend, but different in amplitude. So Case A and Case C are not presented here. At each gap, velocity increases almost linearly to a maximum value and then decreases almost linearly to a minimum value. The maximum value occurs at the points $X_{k},(k=0 \cdots 6)$ at each gap. These points are at the narrowest location along the line. The velocity is larger with respect to a highe pressure differential. These findings on velocity distribution are similar to those by Huang et al. [2].
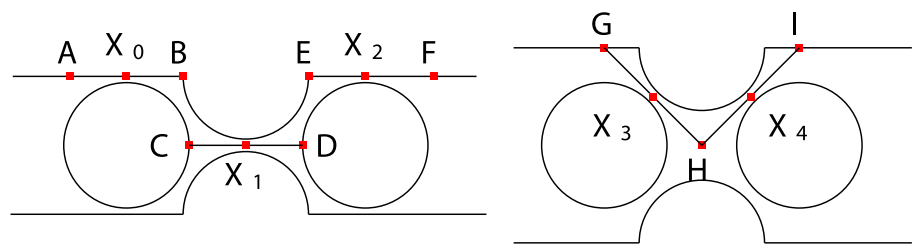

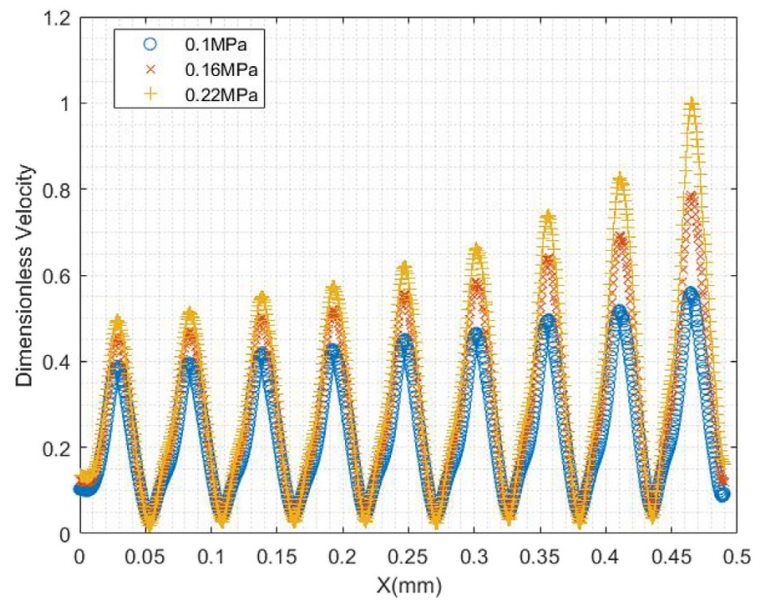

Fig. 9. The velocity distribution at gap centerlines for Case $B_{10}$.

\subsection{Parameters definition}

\subsubsection{Reynolds number}

Reynolds number is a dimensionless quantity to predict the flow pattern in fluid mechanics. In the present study, Reynolds number is used as a quantity to fit the $E u$ correlation, but not to judge whether the flow is laminar or turbulent. It is expressed as the ratio of inertial forces to viscous forces and expressed as

$R e=\frac{\rho U d}{\mu}$

where $U$ is the velocity, and $\mathrm{d}$ is the characteristic length. Researchers have different opinions toward velocity selection, which is an important quantity in calculating Reynolds number. Endres and MÖller proposed four different reference velocities: impinging velocity, percolation velocity, gap velocity and measured gap velocity. The percolation velocity and gap velocity are calculated by proposed formulas, and the other two are obtained by experiments [22]. Žukauskas argued that the main flow velocity should be used as the reference velocity in a model with a tranverse pitch ratio, which is less than 1.25 . Another widely adopted method is described as

$\begin{cases}\frac{u_{\text {main }} S_{T}}{S_{T}-d} & 2 S_{D} \geq S_{T} \\ \frac{u_{\text {main }} S_{T}}{2\left(S_{T}-d\right)} & 2 S_{D}<S_{T}\end{cases}$

where $u_{\text {main }}$ is the main stream velocity. Equation (6) can be derived from the continuity equation. All the velocity in the methods above is constant, which means the kinetic energy keeps constant. This is the reason for the methods mentioned above not appropriate for brush seals. The pressure differential is converted to the kinetic energy when the fluid flows from upstream area to downstream area.

The conventional method of calculating Reynolds number is modified here to calculate Reynolds number in a brush seal. A single bristle and its nearby area are an element domain. Average velocity is adopted due to the dramatic variation of velocity, so the Reynolds number for each bristle can be expressed as

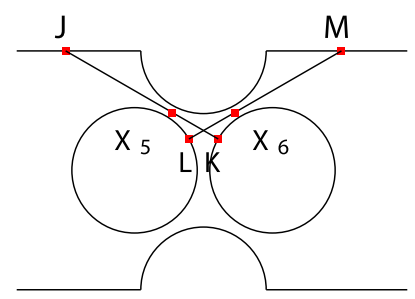

Fig. 8. Schematic diagrams of the centerlines for Case A, Case B and Case C, respectively. 
$R e_{i}=\frac{\rho \bar{U} d}{\mu}$

where subscript $i$ is the bristle sequence, $\bar{U}$ is the average velocity in this gap. Reynolds number for the whole brush seal can be written as follows:

$\operatorname{Re}=\frac{\sum_{i=1}^{N} R e_{i}}{N}$

\subsubsection{Euler number(Eu)}

The $E u$ is, a dimensionless number, introduced to analyze the relationship between pressure reduction and kinetic energy per volume. It is defined as

$E_{u}=\frac{\text { pressureforces }}{\text { inertialforces }}=\frac{(\text { pressure })(\text { area })}{(\text { mass })(\text { acceleration })}=\frac{(\Delta p) L^{2}}{0.5\left(\rho L^{3}\right)\left(U^{2} / L\right)}=\frac{2 \Delta p}{\rho U^{2}}$

where $\Delta p$ is the pressure differential between two successive rows. A constant term 0.5 is multiplied in front of the inertial forces because $0.5 \rho V^{2}$ stands for the kinetic energy, as used by many previous researchers [23-25].

$\mathrm{Eu}$ is the ratio of pressure to kinetic energy. In heat exchanger industry, velocity $U$ in the kinetic energy term is a constant which can be obtained by the methods in Section 3.2.1. Pressure reduction $\Delta p$ can be measured by experiments or extracted from numerical results. But, pressure and kinetic energy selection for a brush seal are different with those for a heat exchanger. Pressure is converted to kinetic energy when the fluid flows a brush seal, indicating that velocity increases and pressure decreases simultaneously. In a brush seal, pressure reduction is a known operation parameter. To express kinetic energy increase, therefore $\mathrm{Eu}$ becomes

$E u=\frac{2 \Delta p}{\rho N\left(U_{e x}^{2}-U_{i n}^{2}\right)}$

where $U_{e x}$ is the maximum velocity at Line $\mathrm{CD}$, and $U_{\text {in }}$ is the velocity along Line $A B$ (As shown in Fig. 5, the flat section of velocity distribution along Line $\mathrm{AB}$ is adopted). The velocity distribution at Line $\mathrm{CD}$ is chosen rather than at the outlet because the kinetic energy dissipates gradually in the exit domain. Also, the overall performance of a brush seal is analyzed rather than the resistance of each bristle. Therefore, the difference of the kinetic energy for the fluid domain is adopted and the gap velocity (as mentioned in Equation (7)) is not used here.

\subsection{Case studies}

Figs. 10-12 demonstrate the trends of $E u$ and leakage of Case A, Case B and Case C, respectively. Among these three cases, Eu decreases

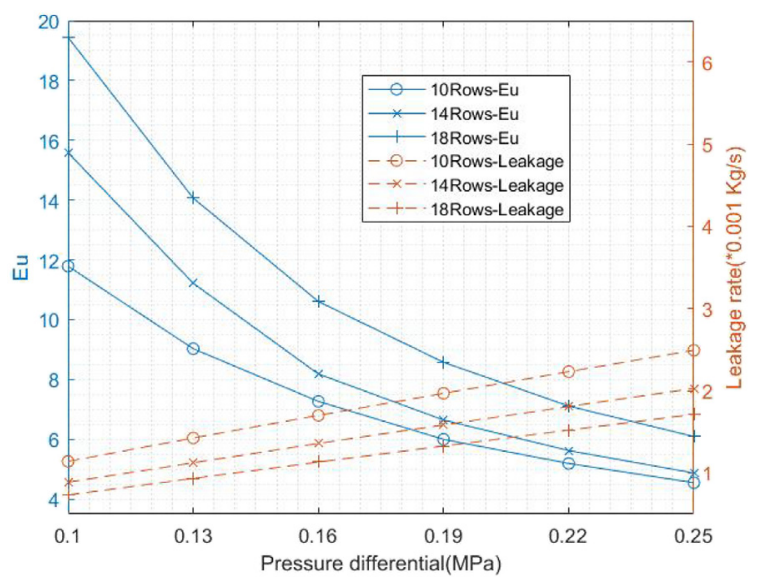

Fig. 10. The $E u$ and leakage for Case A.

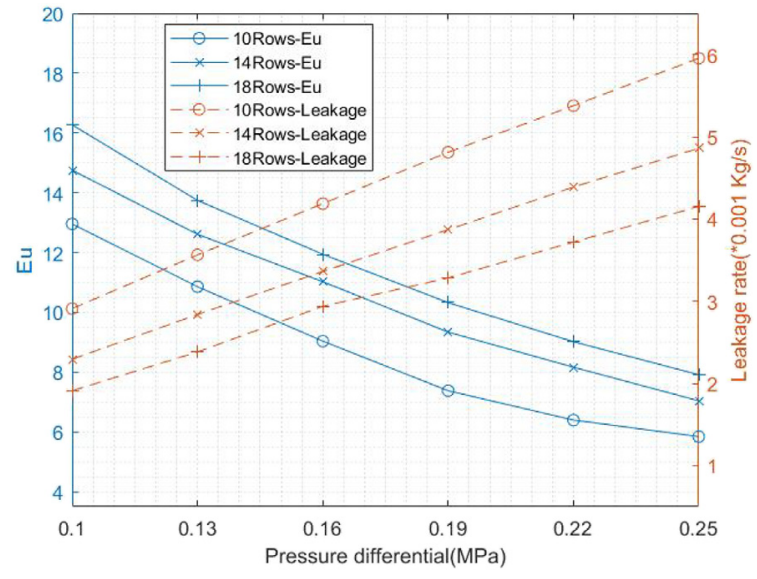

Fig. 11. The $E u$ and leakage for Case B.

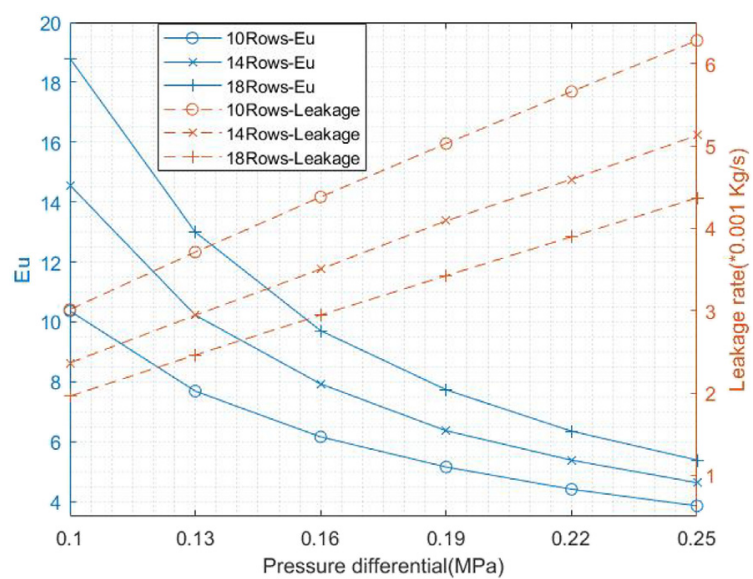

Fig. 12. The $E u$ and leakage for Case C.

with the increase of pressure differentials, indicating that the resistance becomes weak at a high pressure differential. $E u$ becomes larger if a brush seal contained more rows which indicates that adding rows to a brush seal can strengthen its resistance. Another common observation among three cases has been found is that $E u$ decline less gradually at a higher pressure differential than at a lower pressure differential, which can be proved by slopes. Tables 3-5 presents the slope between two successive points for Case A, Case B and Case C, respectively. There is an increase in slopes from a small pressure to a large pressure. All three cases present the same trend and this indicates that the resistant capability becomes gradually weak and approaches a fixed value with the increase of pressure differential, suggesting that the resistance of a brush seal is limited. If a brush seal is subjected to a higher pressure differential, much more pressure energy will convert into kinetic energy, suggesting that fluid flee away at a high velocity. An approximate $E u$ value should be chosen based on experience.

The effect of geometric arrangement on $E u$ is demonstrated in Table 6 . Sign $>$ in Table 6 denotes the resistance is stronger than the

Table 3

The slope between two successive points for Case A.

\begin{tabular}{llllll}
$\begin{array}{l}\text { Pressure Range } \\
\text { (MPa) }\end{array}$ & $0.1-0.13$ & $0.13-0.16$ & $0.16-0.19$ & $0.19-0.22$ & $0.22-0.25$ \\
\cline { 1 - 4 } Row number & & & & & \\
\hline 10Rows & -92 & -58.767 & -42.3 & -26.933 & -17.466 \\
14Rows & -145.67 & -101.13 & -51.37 & -34.07 & -25 \\
18Rows & -179.2 & -115.33 & -67.8 & -48.8 & -33.97
\end{tabular}


Table 4

The slope between two successive points for Case B.

\begin{tabular}{|c|c|c|c|c|c|}
\hline $\begin{array}{l}\text { Pressure Range } \\
(\mathrm{MPa})\end{array}$ & $0.1-0.13$ & $0.13-0.16$ & $0.16-0.19$ & $0.19-0.22$ & $0.22-0.25$ \\
\hline \multicolumn{6}{|l|}{ Row number } \\
\hline 10Rows & -70.053 & -60.686 & -55.488 & -32.55 & -18.431 \\
\hline 14Rows & -71.507 & -52.897 & -56.183 & -39.179 & -37.466 \\
\hline 18Rows & -84.19 & -60.116 & -53.931 & -43.58 & -36.82 \\
\hline
\end{tabular}

Table 5

The slope between two successive points for Case C.

\begin{tabular}{llllll}
\hline $\begin{array}{l}\text { Pressure Range } \\
(\mathrm{MPa})\end{array}$ & $0.1-0.13$ & $0.13-0.16$ & $0.16-0.19$ & $0.19-0.22$ & $0.22-0.25$ \\
\cline { 1 - 5 } Row number & & & & & \\
\hline 10Rows & -89.245 & -50.840 & -33.564 & -24.778 & -18.535 \\
14Rows & -144.673 & -76.686 & -51.874 & -32.853 & -25.05 \\
18 Rows & -193.325 & -109.901 & -65.756 & -45.917 & -32.239 \\
\hline
\end{tabular}

other case. As for a 10-row brush seal, Case B is the most resistant among three cases. For a 14-row brush seal, Case B has the largest resistance at the whole pressure differentials except for $0.1 \mathrm{MPa}$. For a 18row brush seal, Case B is the most resistant among three cases at all the pressure differentials except for 0.1 MPa and 0.13 MPa. Generally, what can be concluded is that Case B is the most resistant. By comparing the $\mathrm{Eu}$ of Case A and Case C in Figs. 10-12, the discrepancy between Case A and Case $\mathrm{C}$ is far less than that between Case B and Case A or Case B and Case $\mathrm{C}$ in most cases, indicating that Case $\mathrm{A}$ and Case $\mathrm{C}$ present similar resistance.

It's hard to define a geometric parameter $\left(S_{T}\right.$ and $\left.S_{L}\right)$ which is most influential on $E u$. Case A has the shortest $S_{T}$ but the longest $S_{L}$. Case C has the shortest $S_{L}$ but the longest $S_{T}$. The difference of $E u$ between Case A and Case C is not obvious. $S_{T}$ and $S_{L}$ of Case B are the compromise between Case A and Case C. And Case B is the most resistant among the three cases. Rather than find which geometric parameter is the most influential on $E u$, the present paper analyzes the correlations between $E u$ and geometric parameters, which are presented in Section 4.

Leakage is an important criterion for judging a seal. For all cases, leakage increases almost linearly with the increase of pressure differential. Leakage of Case A is far less than that of Case B and Case C. For Case $A_{10}^{0.25}$, leakage is about $2.5 \times 10^{-3} \mathrm{~kg} / \mathrm{s}$. For Case $B_{10}^{0.25}$ and $C_{10}^{0.25}$, leakage are approximately $6.0 \times 10^{-3} \mathrm{~kg} / \mathrm{s}$ and $6.4 \times 10^{-3} \mathrm{~kg} / \mathrm{s}$, respectively. Case B has the largest resistance while Case A has the least leakage among three cases. The different trend between leakage and aerodynamic resistance indicates that leakage is not fully equivalent to resistance. Resistance is used to express the energy loss in a brush seal while leakage is obtained at the outlet. For a brush seal, the ideal conditions are low leakage and high energy loss.

\section{Multiple regression}

Based on the numerical results above, multiple regressions between
$E u$ and geometric parameters are obtained. A common correlation for $E u$ is expressed as follows [26].

$E u=f\left(R e, \frac{S_{T}}{d}, \frac{S_{L}}{d}, N\right)$

Žukauskas proposed that $E u$ across a tube banks model is proportional to the number of rows [27]. For practical calculations, the exponential correlation form is assumed

$E u 1=C_{1} \operatorname{Re}^{C_{2}}\left(\frac{S_{T}}{d}\right)^{C_{3}}\left(\frac{S_{L}}{d}\right)^{C_{4}} N$

This paper proposes the correlation with a minor modification of the work by Žukauskas

$E u 2=C_{1} \operatorname{Re}^{C_{2}}\left(\frac{S_{T}}{d}\right)^{C_{3}}\left(\frac{S_{L}}{d}\right)^{C_{4}} N^{C_{5}}$

where coefficients $C_{1}, C_{2}, C_{3}, C_{4}, C_{5}$ are determined by the method of multiple regression. The final correlations are as follows

$E u 1=27.648 R e^{-0.728}\left(\frac{S_{T}}{d}\right)^{0.136}\left(\frac{S_{L}}{d}\right)^{0.29} N$

$E u 2=2429.923 R e^{-0.981}\left(\frac{S_{T}}{d}\right)^{-0.065}\left(\frac{S_{L}}{d}\right)^{0.094} N^{-0.165}$

The residual square $\left(R^{2}\right)$ can quantify how well a correlation fits the data. However, an intrinsic drawback of this method is that $R^{2}$ will always increase if a new predictor is added. The adjusted residualsquare $\left(R_{a d j}^{2}\right)$ can be used to determine how well terms fit the data. The $R_{a d j}^{2}$ is expressed as follows

$R_{\text {adj }}^{2}=1-\left[\frac{\left(1-R^{2}\right)(n-1)}{n-k-1}\right]$

where $n$ is the number of points in a series of data, $k$ is the number of independent regressors. The $R_{a d j}^{2}$ is 0.985 for Eu2(Equation (15)) and 0.654 for Eu1(Equation (14)). Therefore, Eu2 fits the data better than $\mathrm{Eu} 1$.

\section{Conclusion}

The present study analyzed the resistance in the tube banks model of brush seals by adopting $E u$, which has been widely used in the heat exchanger industry. Three different geometric arrangements containing different rows subjected to six pressure differentials were used as the objects. The Reynolds number and Euler number equations were updated according to brush seal working conditions. The present study demonstrats five important findings:

1. Velocity was a constant in the upstream region along Line $\mathrm{AB}$. Velocity distribution along Line $\mathrm{CD}$ was symmetric for part of cases and asymmetric for the other cases. At each gap, velocity increased linearly from the beginning of the gap to the middle and then decreased linearly to the end of the gap.

2. A Reynolds number calculation was proposed based on velocity distribution along gap centerlines, which was applicable for a brush seal subjected to the pressure differential. A conventional $E u$ was modified to analyze its intrinsic aerodynamic resistance of a brush

Table 6

The comparsion of $E u$ among three cases at different rows and pressure differentials.

\begin{tabular}{|c|c|c|c|c|c|c|}
\hline Pressure Differential (MPa) & 0.1 & 0.13 & 0.16 & 0.19 & 0.22 & 0.25 \\
\hline \multicolumn{7}{|l|}{ Row number } \\
\hline 10 & $\mathrm{~B}>\mathrm{C}>\mathrm{A}$ & $\mathrm{B}>\mathrm{C}>\mathrm{A}$ & $\mathrm{B}>\mathrm{C}>\mathrm{A}$ & $\mathrm{B}>\mathrm{C}>\mathrm{A}$ & $\mathrm{B}>\mathrm{C}>\mathrm{A}$ & $\mathrm{B}>\mathrm{C}>\mathrm{A}$ \\
\hline 14 & $\mathrm{~A}>\mathrm{B}>\mathrm{C}$ & $\mathrm{B}>\mathrm{A}>\mathrm{C}$ & $\mathrm{B}>\mathrm{A}>\mathrm{C}$ & $\mathrm{B}>\mathrm{A}>\mathrm{C}$ & $\mathrm{B}>\mathrm{A}>\mathrm{C}$ & $\mathrm{B}>\mathrm{A}>\mathrm{C}$ \\
\hline 18 & $\mathrm{~A}>\mathrm{C}>\mathrm{B}$ & $\mathrm{A}>\mathrm{B}>\mathrm{C}$ & $\mathrm{B}>\mathrm{A}>\mathrm{C}$ & $\mathrm{B}>\mathrm{A}>\mathrm{C}$ & $\mathrm{B}>\mathrm{A}>\mathrm{C}$ & $\mathrm{B}>\mathrm{A}>\mathrm{C}$ \\
\hline
\end{tabular}


seal.

3. $E u$ decreased less gradually with the increase of pressure differentials, indicating that there may exist a minimum $E u$ value for each case. Case B had the largest $E u$ among three cases.

4. A high resistance was not equivalent to low leakage. The $E u$ was interpreted by energy loss while leakage is a quantity measured at the outlet.

5. Two different fitted correlations were achieved between $E u$ and geometric parameters. The correlation that was exponential to the numbers of rows fit better than that which is proportional to the numbers of rows.

\section{Acknowledgments}

The current research has been supported by China Scholarship Council (granted no.201608740003) and National Natural Science Foundation of China (granted no.51765024).

\section{Nomenclature}

N

row number

$p \quad$ pressure $(\mathrm{Pa})$

$\rho \quad$ density of air $\left(\mathrm{Kg} / \mathrm{m}^{3}\right)$

$S_{D} \quad$ diagnoal pitch $(\mathrm{mm})$

$S_{L} \quad$ longitual pitch $(\mathrm{mm})$

$S_{T} \quad$ transverse pitch $(\mathrm{mm})$

$d \quad$ diameter of brislte $(\mathrm{mm})$

$\theta \quad$ angle $\left(^{\circ}\right)$

$\delta \quad$ gap between two bristle $(\mathrm{mm})$

$u_{i}, u_{j} \quad$ velocity component in the $i$ and $j$ direction $(\mathrm{mm})$

$u_{\text {main }} \quad$ main stream velocity $(\mathrm{mm} / \mathrm{s})$

$U \quad$ velocity $(\mathrm{mm} / \mathrm{s})$

$\bar{U} \quad$ average velocity $(\mathrm{mm} / \mathrm{s})$

$u_{e x} \quad$ exit velocity $(\mathrm{mm} / \mathrm{s})$

$u_{\text {in }} \quad$ inlet velocity $(\mathrm{mm} / \mathrm{s})$

$R e_{i} \quad$ located Reynolds number

$R_{e} \quad$ Reynolds number

$E_{u} \quad$ Euler number

$C_{i}(i=1,2,3,4,5) \quad$ coefficients

$R_{a d j}^{2} \quad$ adjusted residual square

\section{References}

[1] Dai W, Liu Y. Numerical simulation of fluid flow across compact staggered tube array. Manuf Autom 2011;33(2):107-10. http://dx.doi.org/10.3969/j.issn.10090134.2011.36.

[2] Huang S, Suo S, Li Y, Yang J, Liu S, Wang Y. Flow in brush seals based on a 2-d staggered tube bundle model. J Tsinghua Univ (Sci Technol) 2016;56(2):160-6. http://dx.doi.org/10.16511/j.cnki.qhdxxb.2015.22.005.
[3] Tan Y, Liu M, Kang Y, Wang X, Liu J. Investigation into brush seal vortex separation point based on 2d staggered tube bundle model. J Drain Irrigat Mach 2017(7):602-8. http://dx.doi.org/10.3969/j.issn.1674-8530.16.0314.

[4] Wang K, Yang Y, Suo S, Jiang J. Analysis of flows of two-stage brush seals and research on configuration of system parameters. Lubr Eng 2017;42(10):43-7.

[5] Jin Y, Tang G-H, He Y-L, Tao W-Q. Parametric study and field synergy principle analysis of h-type finned tube bank with 10 rows. Int J Heat Mass Tran 2013;60:241-51. http://dx.doi.org/10.1016/j.ijheatmasstransfer.2012.11.043.

[6] Chen H, Wang Y, Zhao Q, Ma H, Li Y, Chen Z. Experimental investigation of heat transfer and pressure drop characteristics of h-type finned tube banks. Energies 2014;7(11):7094-104. http://dx.doi.org/10.3390/en7117094.

[7] Kuntysh V, Dudarev V, Sukhotskii A. Experimental study of aerodynamic resistance of staggered equilateral banks of tubes with spiral aluminum fins. Chem Petrol Eng 2015;51(5-6):373-8. http://dx.doi.org/10.1007/s10556-015-0054-8.

[8] Sun D, Liu N, Hu G, Ai Y, Wang K. Fluid-structure interaction investigation on the flow field and mechanical characteristics in brush seals with bristle deflections. J Aero Power 2016;31(10):2544-53. http://dx.doi.org/10.13224/j.cnki.jasp.2016. 10.031 .

[9] Akşit MF. Brush seals and common issues in brush seal applications. NATO; 2012.

[10] Zhu Z. Design and application of a brush seal. Gas Turbine Technol 2005;18(3):68-72.

[11] Chupp RE, Hendricks RC, Lattime SB, Steinetz BM. Sealing in turbomachinery. J Propul Power 2006;22(2):313-49. http://dx.doi.org/10.2514/1.17778.

[12] Dogu Y, Aksit MF. Brush seal temperature distribution analysis. J Eng Gas Turbines Power 2006;128(3):599-609. http://dx.doi.org/10.1115/1.2135817.

[13] Pekris MJ, Franceschini G, Gillespie DR. Effect of geometric changes in an idealised contacting brush seal bristle pack on typical key performance measures, in: ASME 2011 Turbo Expo: turbine Technical Conference and Exposition. Am Soc Mech Eng 2011:999-1010.

[14] Zhang Y, Wang Y, Yan X, Li J. Investigations on the leakage and heat transfer characteristics of brush seal part 1: leakage characteristics. J Eng Thermophys 2017;38(3):482-9.

[15] Zdravkovich M. Flow around circular cylinders. Fundamentals 1997;1.

[16] A. Fluent, 12.0 tutorial guide, Ansys Inc.

[17] F. Fluent, 6.3 users guide, Fluent Inc.

[18] Dogu Y, Aksit MF. Effects of geometry on brush seal pressure and flow fields-part I: front plate configurations. J Turbomach 2006;128(2):367-78.

[19] Bayley F, Long C. A combined experimental and theoretical study of flow and pressure distributions in a brush seal. ASME 1992 international gas turbine and aeroengine congress and exposition American Society of Mechanical Engineers; 1992. http://dx.doi.org/10.1115/92-GT-355. V001T01A120-V001T01A120.

[20] Sumner D, Wong S, Price S, PaĨdoussis M. Fluid behaviour of side-by-side circular cylinders in steady cross-flow. J Fluid Struct 1999;13(3):309-38. http://dx.doi.org/ 10.1006/jfls.1999.0205.

[21] Olinto CR, Indrusiak MLS, Endres LAM, Mller SV. Experimental study of the characteristics of the flow in the first rows of tube banks. Nucl Eng Des 2009;239(10):2022-34. http://dx.doi.org/10.1016/j.nucengdes.2009.05.017.

[22] Endres LAM, Möller SV. Looking for correct dimensionless parameters for tube-bank flow analysis. J Fluid Struct 2001;15(5):737-50.

[23] Singh S, Sørensen K, Condra T. Investigation of material efficient fin patterns for cost-effective operation of fin and tube heat exchanger. Appl Therm Eng 2017;126:903-14. http://dx.doi.org/10.1016/j.applthermaleng.2017.08.010.

[24] Chen H, Wang Y, Zhao Q, Ma H, Li Y, Chen Z. Experimental investigation of heat transfer and pressure drop characteristics of h-type finned tube banks. Energies 2014;7(11):7094-104. http://dx.doi.org/10.3390/en7117094.

[25] Marr YN. Investigation of the aerodynamic resistance of a staggered bank of finned tubes. Chem Petrol Eng 1969;5(10):781-3. http://dx.doi.org/10.1007/ BF01153170.

[26] Žukauskas A, Ulinskas R. Efficiency parameters for heat transfer in tube banks. Heat Tran Eng 1985;6(1):19-25.

[27] Žukauskas A. Heat transfer from tubes in crossflow. Adv Heat Tran 1972;8:93-160. 